\title{
Adrenocortical Carcinoma with Inferior Vena Cava Thrombus extending up to Right Atrium: Evaluation and Management
}

\author{
${ }^{1}$ Sureka S Kumar, ${ }^{2}$ Sabaretnam Mayilvaganan, ${ }^{3}$ Gauranga Majumdar, ${ }^{4}$ Surendra K Agarwal \\ ${ }^{5}$ Prabhat Tewari, ${ }^{6}$ Amit Agarwal
}

\section{ABSTRACT}

Malignancy of the adrenal cortex is uncommon but aggressive and grows rapidly and metastasizes to the liver, lungs, kidneys, and bones. Very rarely, tumor thrombus may extend through via adrenal vein to the inferior vena cava (IVC) and even may extend up to the right atrium. Such a scenario is rare and possesses a real surgical challenge. We present a case of right adrenocortical tumor with IVC thrombus extending until the right atrium. The patient successfully underwent right adrenalectomy with nephrectomy and retroperitoneal lymphadenectomy and removal of its intravascular extension with the assistance of cardiopulmonary bypass and intraoperative transesophageal echocardiography.

${ }^{1,2}$ Assistant Professor, ${ }^{3-6}$ Professor

${ }^{1}$ Department of Urology, Sanjay Gandhi Postgraduate Institute of Medical Sciences, Lucknow, Uttar Pradesh, India

${ }^{2,6}$ Department of Endocrine Surgery, Sanjay Gandhi Postgraduate Institute of Medical Sciences, Lucknow, Uttar Pradesh, India

3,4 Department of CVTS, Sanjay Gandhi Postgraduate Institute of Medical Sciences, Lucknow, Uttar Pradesh, India

${ }^{5}$ Department of Anesthesia, Sanjay Gandhi Postgraduate Institute of Medical Sciences, Lucknow, Uttar Pradesh, India

Corresponding Author: Sureka S Kumar, Assistant Professor Department of Urology, Sanjay Gandhi Postgraduate Institute of Medical Sciences, Lucknow, Uttar Pradesh, India, Phone: +918004904749, e-mail: drsksureka@gmail.com
Keywords: Adrenocortical carcinoma, Contrast enhanced computed tomography, Inferior vena cava thrombus, Ultrasonography and doppler.

How to cite this article: Kumar SS, Mayilvaganan S, Majumdar G, Agarwal SK, Tewari P, Agarwal A. Adrenocortical Carcinoma with Inferior Vena Cava Thrombus extending up to Right Atrium: Evaluation and Management. World J Endoc Surg 2017;9(3):118-119.

Source of support: Nil

Conflict of interest: None

\section{CASE REPORT}

A 32-year-old lady was evaluated for right flank pain, weight loss, and generalized weakness and diagnosed as a case of right-sided large adrenal tumor, with tumor thrombus extending to right atrium via right adrenal vein and IVC by means of contrast-enhanced computed tomography (CECT) abdomen (Figs 1A and B) and thorax and magnetic resonance angiography. An echocardiographic evaluation confirmed the presence of the tumor thrombus in the right atrium. The patient underwent right adrenalectomy with nephrectomy and retroperitoneal lymphadenectomy and removal of its intravascular extension with the assistance of cardiopulmonary bypass (Figs 1C and D) and intraoperative transesophageal
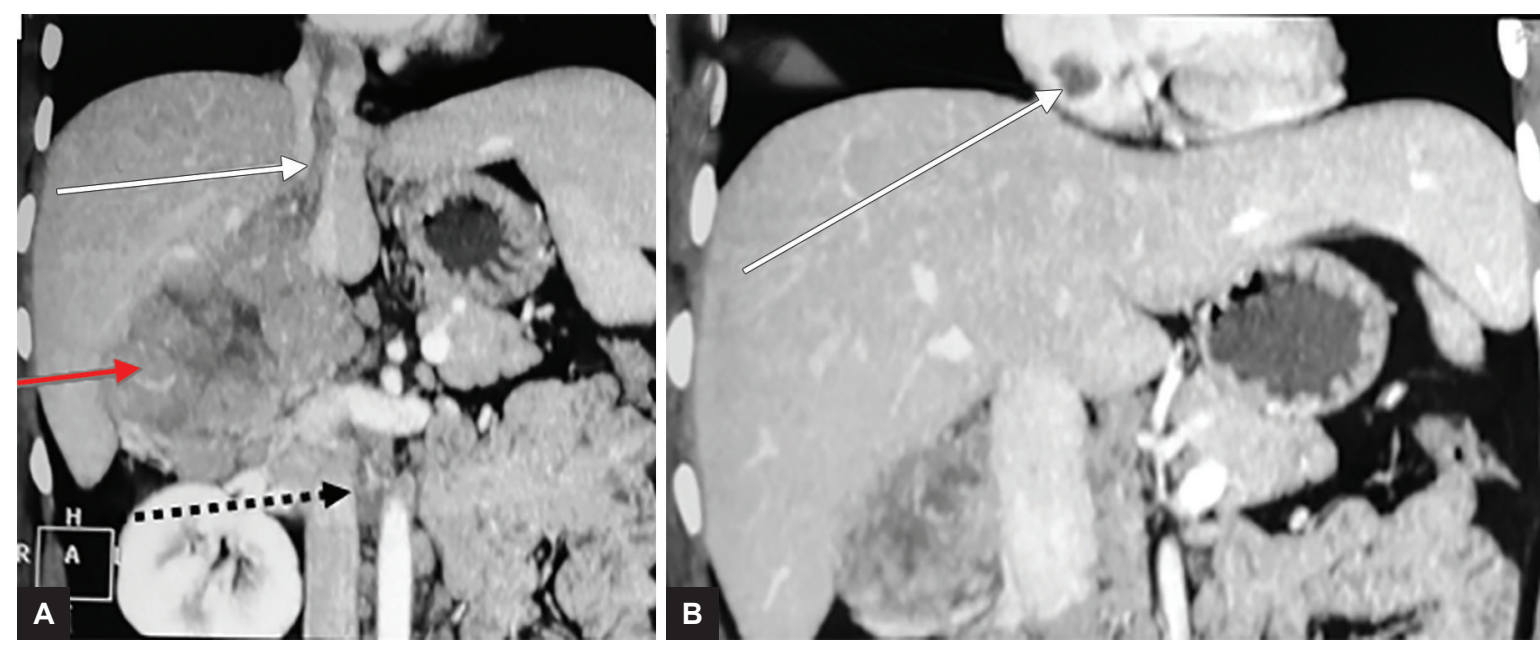

Figs $1 \mathrm{~A}$ and $\mathrm{B}$ : A CECT scan showing right ACC with IVC thrombus extending to right atrium. (A) White solid arrow, IVC thrombus; red arrow, right adrenal mass; dashed black arrow, interaortocaval lymph nodes; (B) White arrow showing thrombus extending to right atrium

(Cont'd...) 

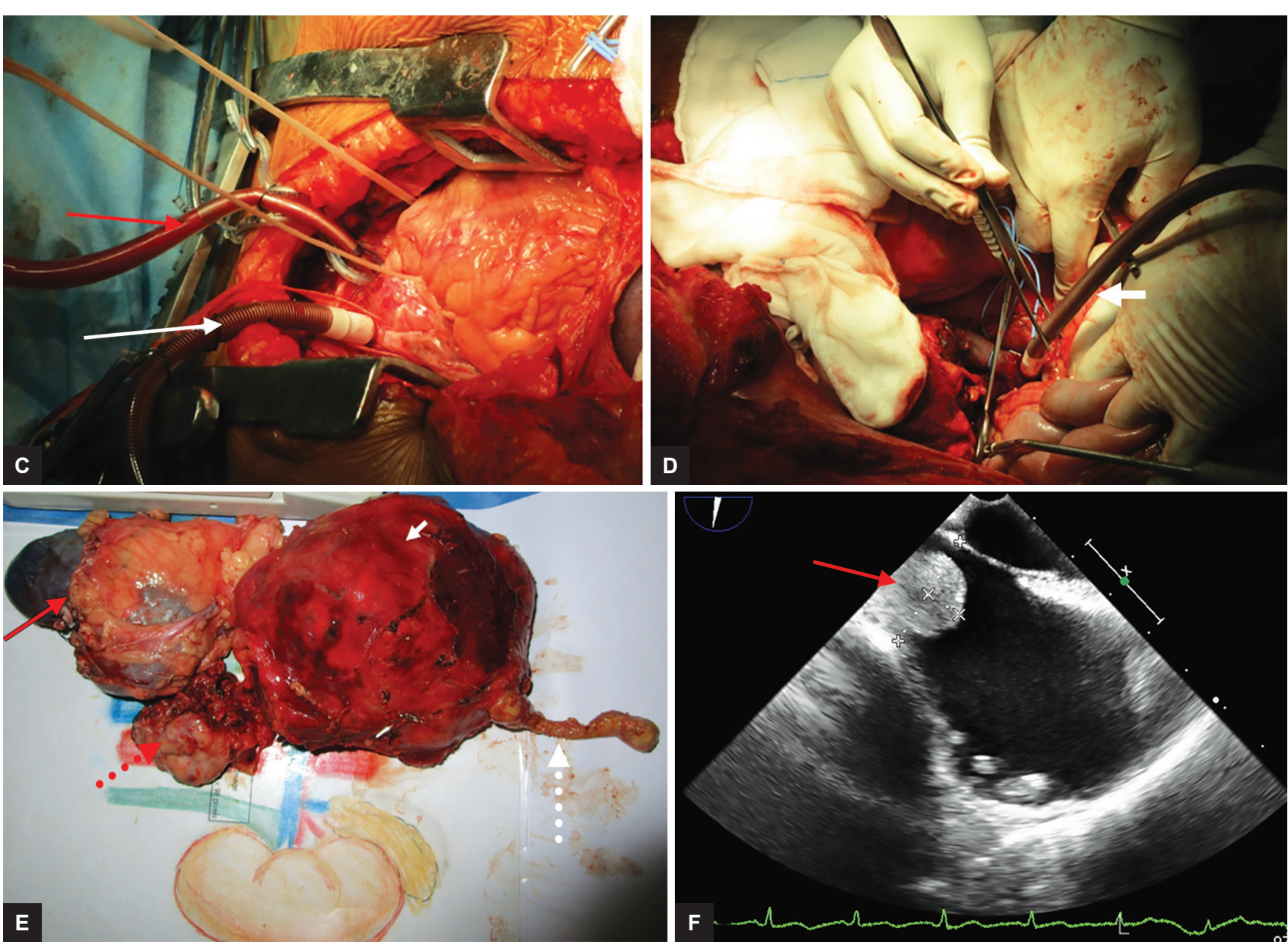

Figs 1C to F: (C) Red, solid aortic cannulation; white arrow, SVC cannulation; (D) White arrow, IVC cannulation; (E) Intraoperative transesophageal USG showing thrombus in right atrium; and (F) Red solid arrow, Specimen showing right kidney; white solid arrow, adrenal mass; white dashed arrow, thrombus; and red dashed arrow, retroperitoneal lymph nodes

echocardiography (Fig. 1E). Specimen was checked to confirm complete removal of thrombus with convex upper edge (Fig. 1F). Postoperative recovery was uneventful and she was discharged on postoperative day 8. Histopathology confirmed adrenocortical carcinoma (ACC). Follow-up at 3 and 6 months with ultrasonography (USG) of whole abdomen and Doppler and CECT scan was normal.

\section{CONCLUSION}

The ACC with tumor thrombus extending into the IVC or even up to the right atrium does not represent a contraindication to surgery, and curative resection is feasible. Preoperative optimal surgical planning and execution of each and every step carefully in a multidisciplinary setting is the key. 